\title{
LES FRAYÈRES POTENTIELLES DE L'ESTURGEON EUROPÉEN ACIPENSER STURIO L. 1758 DANS LE BASSIN GARONNE-DORDOGNE. MÉTHODES D'INVESTIGATION, ÉTAT ACTUEL ET PERSPECTIVES.
}

\author{
S. JEGO, C. GAZEAU, P. JATTEAU*, P. ELIE, E. ROCHARD \\ Cemagref, Groupement de Bordeaux, Unité Ressources Aquatiques Continentales, \\ 33612 CESTAS Cedex, France. \\ *E-mail : philippe.jatteau@ cemagref.fr
}

\section{RÉSUMÉ}

Dans le cadre du programme de restauration de l'esturgeon européen Acipenser sturio L. 1758, nous avons recensé et caractérisé l'ensemble des frayères potentielles de cette espèce sur les fleuves Garonne et Dordogne. L'objectif de ce travail était de réaliser un premier bilan sur l'état actuel de ces zones et d'évaluer leur possible utilisation comme frayère.

Avant ce travail, les seules données disponibles étaient constituées par une liste de frayères et de zones de pêche de géniteurs répertoriées en 1952 et 1980 par enquêtes auprès des pêcheurs. Une analyse des données publiées sur les autres espèces d'esturgeons a permis de cerner les principales caractéristiques physiques d'une frayère d'esturgeons. En croisant les informations issues de ces 2 sources, historique et bibliographique, nous avons retenu une liste de sites qui ont ensuite été caractérisés lors de campagnes sur le terrain. Cette caractérisation a porté sur la bathymétrie, le courant, la nature du substrat, la position dans le paysage et les impacts anthropiques immédiats.

Les résultats de cette étude ont montré que la majorité des 28 sites étudiés répondait aux caractéristiques d'une frayère d'esturgeon en terme de courant, profondeur et substrat. Ils pouvaient donc être considérés comme frayères potentielles et leur capacité d'accueil était suffisante à moyen terme. Néanmoins il apparaît nécessaire de protéger réglementairement l'ensemble de ces sites afin de les soustraire aux impacts négatifs d'activité anthropiques.

\section{SPAWNING GROUNDS AVAILABLE FOR THE EUROPEAN STURGEON ACIPENSER STURIO L. 1758 IN THE GARONNE-DORDOGNE BASIN. METHODS USED, PRESENT STATUS AND PROSPECTS.}

\section{ABSTRACT}

Within the framework of the programme of restoration of the European sturgeon Acipenser sturio L. 1758, we listed and characterized the whole of the potential spawning 
grounds of this species in the Garonne and Dordogne rivers. The objective of this work was to carry out a first assessment on the current status of these zones and to evaluate their possible use as spawning ground. Before this work, the only available data consisted of a list of spawning grounds and spawner fishing zones indexed in 1952 and 1980 by investigations of the fishermen. An analysis of the data published on the other species of sturgeons made possible to determine the main physical characteristics of a spawning ground of sturgeons. By crossing information resulting from these 2 sources, historical and bibliographical, we retained a list of sites which were characterized at the time of field campaigns. This characterization related to bathymetry, current speed, nature of the substrate, position in the landscape and immediate anthropic impacts.

The results of this study showed that the majority of the 28 studied sites met the characteristics of a spawning ground of sturgeons in term of current, depth and substrate. They could thus be regarded as potential spawning grounds, and their yield capacity potential was sufficient in the mid-term. Nevertheless, it appears necessary to protect the whole sites according to the regulations in order to withdraw them from the negative impacts of anthropic activity.

\section{INTRODUCTION}

Les esturgeons sont des poissons Chondrostéens dont l'aire de répartition est limitée à l'hémisphère nord. Les 24 espèces d'esturgeons se reproduisent toutes en eau douce, mais certaines sont strictement dulçaquicoles, et d'autres sont amphihalines (ROCHARD et al., 1990). L'esturgeon européen, Acipenser sturio L. 1758, fait partie de cette dernière catégorie. C'est une espèce en danger d'extinction et protégée en France depuis 1982 et à l'échelle européenne depuis 1998 (annexe II de la Convention de Berne). II semble très probable que la population du bassin versant Gironde-Garonne-Dordogne soit la seule encore en fonctionnement (ROCHARD et al., 1997). Des captures de juvéniles de l'année dans le haut estuaire en 1988 et 1994 sont des preuves de reproductions naturelles en Garonne ou en Dordogne (ROCHARD, 1992 ; WILLIOT et al., 1997). Le programme de restauration de l'espèce a reçu le soutien de la Communauté Européenne par l'intermédiaire d'un contrat Life en 1994 (ELIE, 1997). Le succès d'un tel programme et la gestion d'une population fragile nécessitent des actions de recherche prioritaires pour l'identification et la caractérisation des zones d'habitats essentiels pour les différentes écophases (THERRIEN, 1998). Dans le cadre de ce programme une opération particulière a porté sur l'étude des zones de reproduction de l'esturgeon (ELIE et al., 1997 ; JEGO et al., 1997).

Afin d'asseoir la cohérence du programme de restauration, nous avions besoin d'un recensement et d'un diagnostic précis des zones de frayères. Les résultats de cette action avaient pour objectif :

- de déterminer le nombre, la localisation et de décrire les caractéristiques physiques des sites ;

- d'estimer les capacités du milieu en terme de potentiel de reproduction ;

- de réaliser un état de référence pour permettre de suivre l'évolution de ces sites ;

- de proposer des mesures de protection ou de réhabilitation de certains sites.

Les connaissances sur les frayères à Acipenser sturio sont très fragmentaires. Elles reposent sur un inventaire et une description sommaire des zones de pêche de géniteurs 
assimilées à des sites de frai, réalisées à partir d'enquêtes auprès d'anciens pêcheurs (ANONYME, 1980a, 1980b) et d'une étude préliminaire (EPIDOR, 1996). Mais ces zones et leurs abords ont subi pendant de longues années des extractions massives de granulats (TROUVERY et al., 1984 ; CASTELNAUD et al., 1991), qui ont eu pour principales conséquences une modification de la nature du fond et du profil d'écoulement des eaux (ROCHARD et al., 1990). La démarche logique d'identification des sites de reproduction consiste, soit à récupérer les produits de la reproduction (HATIN et al., 1998 ; LA HAYE et CLERMONT, 1996 ; SULAK et CLUGSTON, 1998 ; THIBODEAU, 1997 ; VOTINOV et KAS'YANOV, 1978), soit à suivre le déplacement de géniteurs jusqu'aux frayères (HALL et al., 1991 ; KIEFFER et KINARD, 1996 ; MCKINLEY et al., 1998 ; SCHAFFTER, 1997). Etant donné le faible effectif actuel de géniteurs et la rareté des reproductions naturelles, ce type d'approche nécessiterait d'importants moyens avec d'infimes chances de réussite et n'a donc pas été retenu. Afin d'identifier les zones de frayères, qualifiées de potentielles car aucune observation récente de reproduction naturelle ne permet de valider leur utilisation, nous avons choisi de procéder par analogie. La première étape consiste à analyser les informations bibliographiques dans le domaine afin de dégager les caractéristiques physiques communes aux frayères d'Acipenséridés. En postulant que les frayères d'Acipenser sturio répondent aux mêmes critères, la seconde étape consiste à localiser et à étudier l'ensemble des sites ayant ces propriétés.

\section{Résultats de l'analyse bibliographique}

Les travaux portant sur les frayères d'esturgeon documentent principalement les paramètres en rapport avec leur mode de reproduction de type litho-pélagophile (BALON, 1975), à savoir le substrat, le courant et la profondeur.

Les zones de frayères sont généralement situées dans les parties profondes des fleuves, dans l'extérieur d'un méandre ou près des berges (PRIEGEL et WIRTH, 1971 ; HALL et al., 1991). De nombreux sites de frai sont décrits juste en aval d'un obstacle infranchissable (BUCKLEY et KINARD, 1985 ; SLIVKA et PAVLOV, 1982 ; KEMPINGER, 1988 ; DENG XHONG-LING et XU YUNGAN ZHAO YAN, 1991 ; PARSLEY et BECKMAN, 1994). Les sites en zone de marée dynamique sont rares. KIEFFER et KINARD (1996) mentionnent une frayère fonctionnelle subissant un marnage mais sans inversion de courant. BAIN (1997) décrit des sites de frai d'Acipenser oxyrinchus Mitchill 1815 subissant une inversion de courant. Chez Acipenser sturio, VELEZ SOTO (1951) a signalé des activités de frai en aval d'un barrage, en zone saumâtre, sans donner d'information sur la survie des œufs.

La superficie des frayères est généralement importante, de l'ordre de plusieurs dizaines d'hectares, mais une frayère fonctionnelle de 1 hectare a été décrite chez Acipenser oxyrinchus (SULAK et CLUGSTON, 1998, 1999).

La profondeur des sites de frai montre une grande variabilité entre 4 et $27 \mathrm{~m}$ (PIROGOVSKII et al., 1989 ; TANASIISHUK, 1964 in VLASENKO et al., 1989 ; SOKOLOV et VASIL'EV, 1989 ; PARSLEY et al., 1993 ; HALL et al., 1991 ; KIEFFER et KINARD, 1996 ; MARCHANT et SHUTTERS, 1996).

Seules les frayères d'Acipenser fulvescens Rafinesque 1817 ont une profondeur maximale de l'ordre de $3 \mathrm{~m}$ (PROVOST et al., 1982 ; LECLERC, 1987 ; LAMONTAGNE et GILBERT, 1990). PRIEGEL et WIRTH (1971) et KEMPINGER (1988) mentionnent que le frai se déroule près des berges.

Les vitesses de courant relevées sur les frayères se situent entre 0,5 et $2 \mathrm{~m}^{-1} \mathrm{~s}^{-1}$. Des sites de frai peuvent être localisés dans des zones de légers remous (PRIEGEL et WIRTH, 1971 ; HALL et al., 1991) ou bien dans des zones tourbillonnaires avec inversion de 
courant sur le fond (MCKINLEY et al., 1998 ; SULAK et CLUGSTON, 1998). Dans ce dernier cas le courant au fond est de l'ordre de 0,1 à $0,4 \mathrm{~m} \cdot \mathrm{s}^{-1}$.

L'ensemble des études montre que le substrat des frayères est de nature hétérogène. La granulométrie des éléments s'échelonne selon les sites de $250 \mathrm{~mm}$ pour les blocs à $3 \mathrm{~mm}$ pour le gravier (DENG XHONG-LING et XU YUNGAN ZHAO YAN, 1991; VLASENKO, 1974 ; BUCKLEY et KINARD, 1985 ; KIEFFER et KINARD, 1996 ; MC CABE et TRACY, 1994 ; PARSLEY et al., 1993 ; LA HAYE et al., 1992 ; VOTINOV et KAS'YANOV, 1978). Par contre les substrats plats et uniformes de type limon, argile, sable fin ou roche mère ne sont pas utilisés pour la reproduction (LA HAYE et al., 1992).

Cette analyse de bibliographie appelle 4 remarques:

- les rares données concernant les frayères d'Acipenser sturio (MAGNIN, 1962 ; NINUA, 1976) sont cohérentes avec celles des autres espèces ;

- la majorité des études portent sur des frayères en aval d'un barrage. Les esturgeons étant dans l'impossibilité de gagner des zones plus amont, il semble que ces sites soient des frayères forcées ;

- les études sont généralement focalisées sur les sites de fixation des œufs, mais aussi des sites de regroupements de géniteurs (PRIEGEL et WIRTH, 1971) ;

- les valeurs des paramètres profondeur et courant sont mesurées en différents points remarquables des zones d'étude, variables selon les auteurs, comme le lieu de capture de géniteurs (BUCKLEY et KINARD, 1985 ; KIEFFER et KINARD, 1996) ou le site de fixation des œufs (KEMPINGER, 1988 ; LA HAYE et al., 1992 ; MC CABE et TRACY, 1994 ; PARSLEY et al., 1993 ; SULAK et CLUGSTON, 1998 ; SCHAFFTER, 1997). L'origine des valeurs de vitesse de courant est encore plus hétérogène car elle regroupe des mesures dans la colonne d'eau (BUCKLEY et KINARD, 1985), près du fond (PARSLEY et al., 1993), une moyenne de 3 mesures dans la colonne d'eau (LA HAYE et al., 1992) ou sans localisation précisée (VOTINOV et KAS'YANOV, 1978).

Ces 2 dernières remarques soulignent la difficulté de dégager les principales caractéristiques physiques d'une frayère. Cette notion comprend la zone de dépôt des œufs, ou bien la zone de dépôt des œufs plus les aires de regroupement - repos des géniteurs. Comme cet élément n'est pas toujours précisé dans les études, nous avons considéré que ces 2 zones faisaient partie intégrante de notre modèle. Le modèle théorique de frayère que l'on tente d'établir à partir de cette analyse repose sur les valeurs les plus généralement rencontrées au cours de la période de reproduction. Les caractéristiques de ce modèle sont les suivantes :

- présence d'une zone de profondeur minimale de $5 \mathrm{~m}$;

- substrat de nature hétérogène de granulométrie grossière (gravier, galet, bloc) ;

- vitesse de courant au fond de l'ordre de $0,5 \mathrm{~m} \cdot \mathrm{s}^{-1}$ minimum permettant de disperser les œufs tout en préservant leur fixation dans la zone propice à leur développement ;

- les sites directement en aval d'un obstacle doivent être considérés comme des frayères potentielles. 


\section{MATÉRIEL ET MÉTHODES}

\section{Présentation de la zone d'étude}

La zone d'étude est constituée par la partie aval de la Garonne et de la Dordogne (Figure 1). La limite amont est constituée par les obstacles infranchissables pour l'esturgeon que sont le barrage de Bergerac situé à $211 \mathrm{~km}$ de l'embouchure (altitude $20 \mathrm{~m}$ ) sur la Dordogne et le seuil de Beauregard à $270 \mathrm{~km}$ de l'embouchure (altitude $36 \mathrm{~m}$ ) sur la Garonne. La marée dynamique est sensible jusqu'à Flaujagues sur la Dordogne et Casseuil sur la Garonne. Cette zone de marée dynamique est également caractérisée par la présence d'un bouchon vaseux qui se déplace en fonction des conditions hydrologiques. Sa limite d'intrusion amont peut atteindre celle de la marée dynamique lorsque l'étiage est sévère. Lorsque le courant est trop faible la décantation d'une partie du bouchon vaseux engendre la formation de couches de vase fluide appelée " crème de vase ". La remontée de la crème de vase se fait beaucoup plus en amont sur la Dordogne du fait des plus faibles débits de ce fleuve.

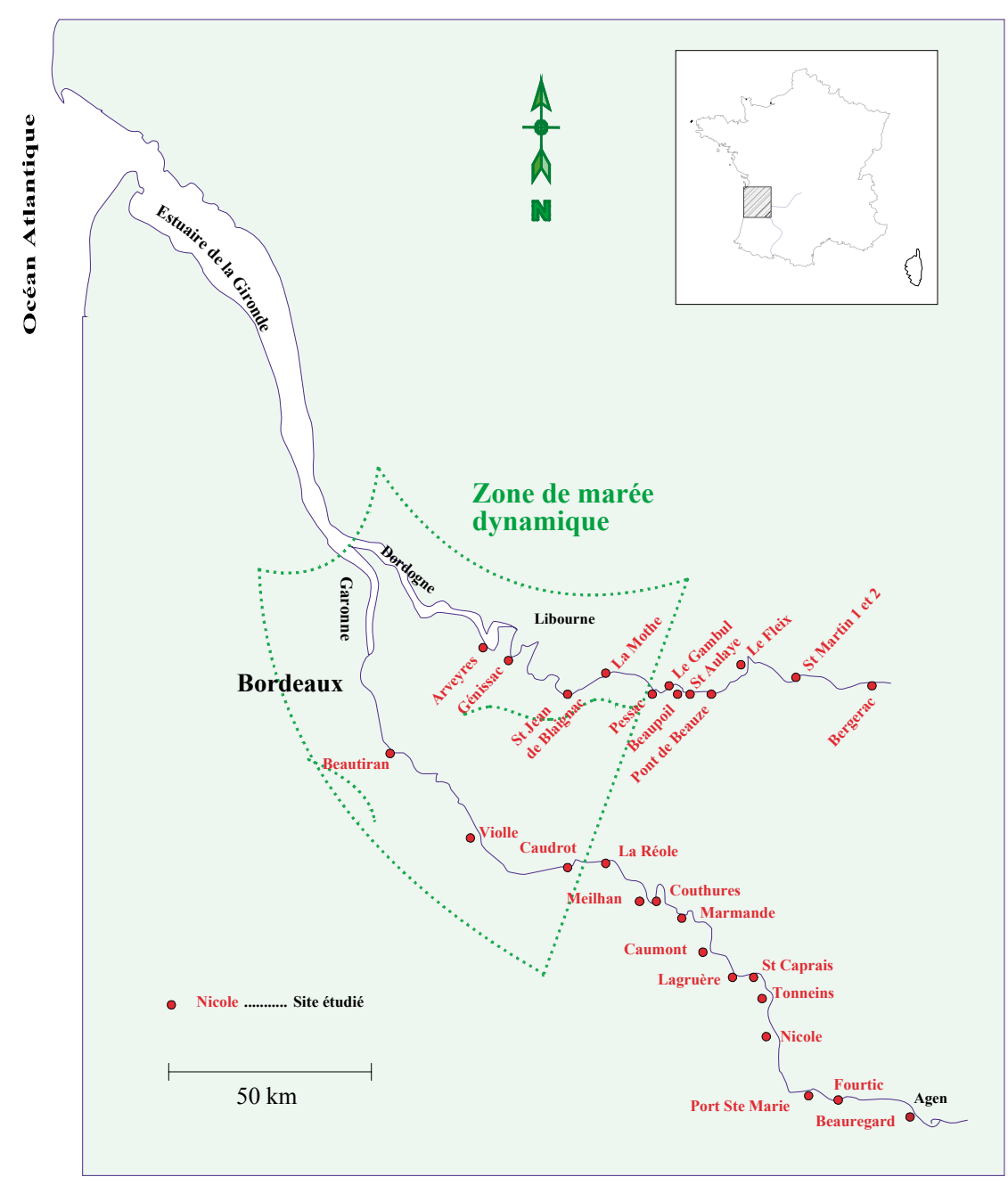

Figure 1

Présentation de la zone d'étude et localisation des sites étudiés.

Figure 1

Presentation of the study area and localization of the studied sites. 
La surface des bassins versants des 2 fleuves est de $71000 \mathrm{~km}^{2}\left(55000 \mathrm{~km}^{2}\right.$ pour la Garonne et $16000 \mathrm{~km}^{2}$ pour la Dordogne). Les débits moyens annuels calculés sur 30 ans sont de $631 \mathrm{~m}^{3} \cdot \mathrm{s}^{-1}$ pour la Garonne et de $338 \mathrm{~m}^{3} \cdot \mathrm{s}^{-1}$ pour la Dordogne.

L'étude s'est déroulée en 1996 du 28 mai au 13 juin pour les sites sur la Dordogne, et du 18 juin au 11 juillet pour les sites sur la Garonne.

\section{Le choix des sites}

A partir du modèle théorique de frayère, nous avons utilisé 2 critères pour la sélection des sites à étudier. Le critère principal est la présence d'une profondeur minimale de $5 \mathrm{~m}$. Ceci peut être déterminé à partir de relevés bathymétriques existants. Cette sélection à partir du critère profondeur couvre des sites historiquement connus de pêche de géniteurs, mais aussi des sites non répertoriés en tant que tels. Cette démarche n'était pas réalisable avec les critères nature du substrat et vitesse de courant du fait du manque de données disponibles.

Ce critère profondeur nous a conduit à identifier 26 sites (Figure 1) :

- sept sites sont dans la zone de marée dynamique. Deux subissent un simple marnage ( 1 sur chaque fleuve), et 5 subissent en plus une inversion de courant ( 3 sur la Dordogne) ;

- en zone strictement fluviale, on dénombre 11 sites en Garonne et 8 en Dordogne ;

En second lieu les 2 zones situées en aval du premier obstacle infranchissable, Bergerac sur la Dordogne et seuil de Beauregard sur la Garonne ont été ajoutées à cette liste, considérant qu'elles pourraient constituer des frayères forcées.

Au total 28 sites ont donc été retenus.

\section{Le protocole de caractérisation}

Sur chaque site, la caractérisation commence par l'enregistrement d'un profil bathymétrique longitudinal amont-aval permettant de repérer les limites de la fosse. Trois profils bathymétriques transversaux sont ensuite enregistrés (Figure 2) :

- 1 profil aval placé sur le radier qui succède à la fosse (transect $A$ ) avec 2 points de mesure situés à $1 / 3$ et à $2 / 3$ de la largeur du lit ;

- 1 profil intermédiaire (transect $B$ ) au niveau de la partie la plus profonde de la fosse, avec 3 points de mesure situés à 1/4, 1/2, 1/4 de la largeur du lit ;

- 1 profil amont (transect C) situé en début de fosse, avec 3 points de mesure répartis comme pour le profil intermédiaire.

Pour chaque point des mesures d'oxygène dissous et de température (oxymètre YSY 51B), sont réalisées en surface et au fond. La vitesse du courant est mesurée en temps réel à 0,2 et 0,8 fois la hauteur d'eau (General Oceanics $2031 \mathrm{H}$ avec boîtier de lecture $2035 \mathrm{MK} 4$ ) avec une précision de $0,1 \mathrm{~m} \cdot \mathrm{s}^{-1}$. Un prélèvement de substrat est réalisé par dragage pendant 15 secondes à l'aide d'une drague de type "Charcot » ayant un maillage carré de $1 \mathrm{~mm}$ de côté. Au point 1B nous avons mesuré en plus la transparence de l'eau (disque de "Secchi » de $30 \mathrm{~cm}$ de diamètre) et nous avons relevé la position GPS de la fosse (récepteur Nav Trac XL). 


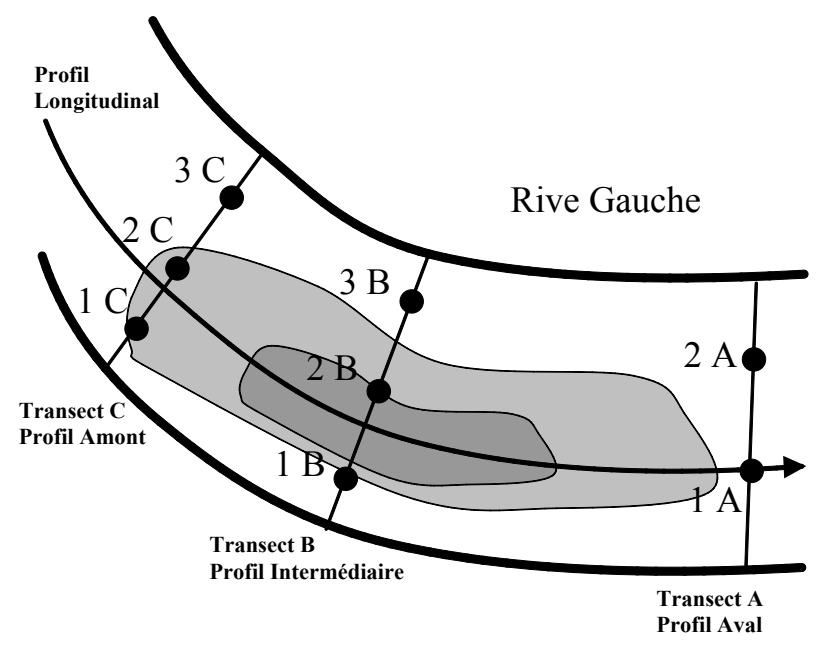

Rive Droite

Figure 2

Situation des points de prélèvements et des profils bathymétriques.

\section{Figure 2}

Localization of the measurement points and the bathymetric profiles.

Les données de débit et de température prévalant durant la période de l'étude ont été obtenues auprès de la Direction Régionale de l'Environnement Aquitaine, Electricité de France et l'association Migrateur Garonne Dordogne.

\section{Traitement des échantillons et des données}

Les échantillons de substrat récoltés ont été passé à travers différents tamis à maille carrée après séchage à l'étuve pour déterminer leur composition en fonction de 6 classes granulométriques (Tableau I).

\section{Tableau I}

Classes granulométriques d'après la norme NF P94-056 (en mm).

\section{Table I}

Granulometric classes following the NF P94-056 standard (in mm).

\begin{tabular}{|c|c|}
\hline Classes granulométriques & Taille des particules \\
\hline limons - argiles & $<0,05$ \\
\hline sables fins & $0,05-0,20$ \\
\hline sables grossiers & $0,20-2,00$ \\
\hline graviers & $2,00-20,00$ \\
\hline cailloux & $20,00-100,00$ \\
\hline blocs & $100,00-250,00$ \\
\hline
\end{tabular}


Deux catégories de substrats identifiées sur le terrain ont été ajoutées :

- la « roche-mère » (lorsque le prélèvement était nul) ;

- la « crème de vase ».

La superficie des sites correspond à la surface de la zone comprise entre les transects amont et aval et présentant une profondeur supérieure ou égale à $4 \mathrm{~m}$.

Une estimation de la vitesse moyenne du courant dans la colonne d'eau au niveau de la fosse est calculée à partir des 2 mesures effectuées (BOVEE, 1986).

\section{RÉSULTATS}

Les débits moyens mensuels sont en légère diminution entre les mois d'avril et de juin, et en forte diminution à partir du mois de juillet (Figure 3).

On constate que la température est en légère augmentation d'avril à mai sur les 2 cours d'eau (Figure 3). A partir de la mi-mai, l'augmentation est rapide pour atteindre environ $10^{\circ} \mathrm{C}$ en un mois.

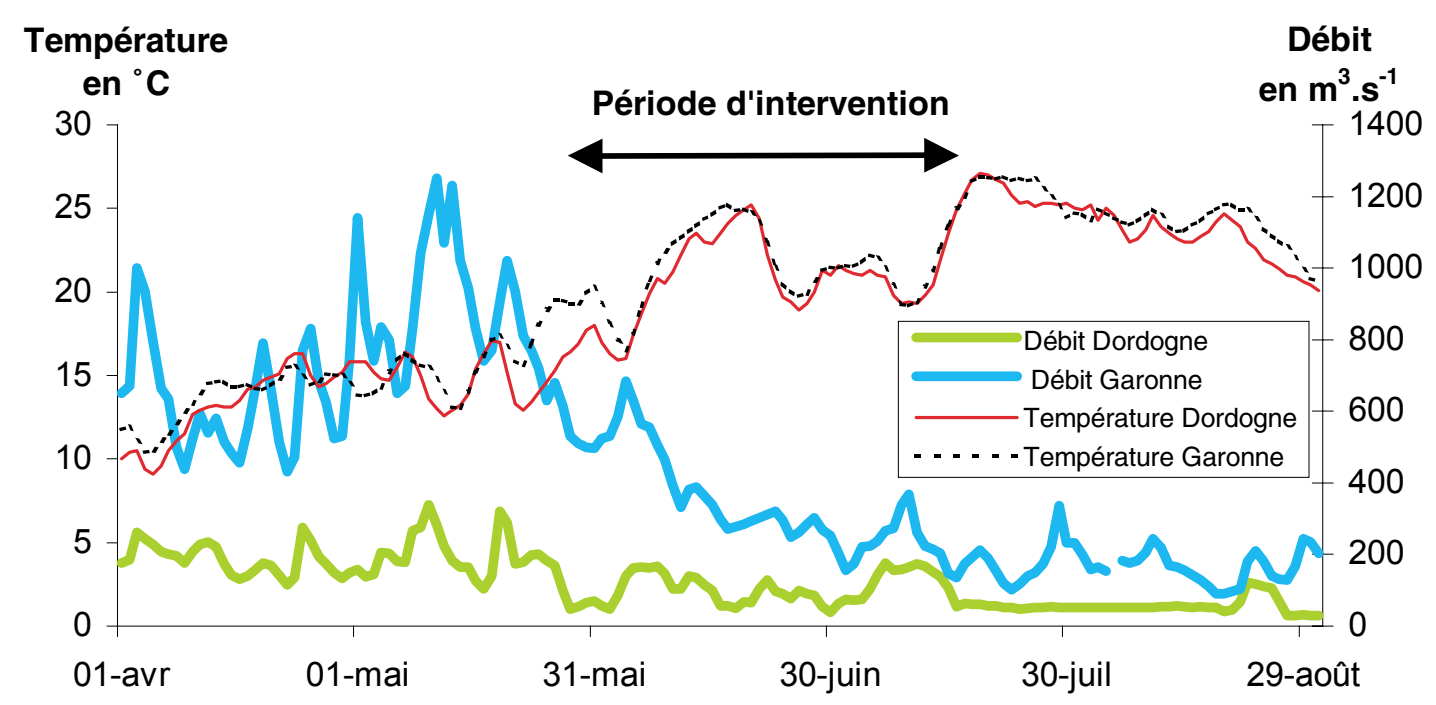

\section{Figure 3}

Température et débit de la Garonne et de la Dordogne en 1996.

\section{Figure 3}

Temperature and flow rate of the Garonne and the Dordogne in 1996. 
Les vitesses moyennes de courant au niveau de la fosse sont regroupées sur la Figure 4. Elles sont toutes inférieures à $1 \mathrm{~m} . \mathrm{s}^{-1}$. Sur 12 sites les vitesses moyennes sont comprises entre 0,3 et $0,5 \mathrm{~m} \cdot \mathrm{s}^{-1}$. Hormis les sites de Bergerac et de Beauregard situés juste en aval d'un barrage, seulement 3 sites ont des vitesse de courant supérieures à $0,5 \mathrm{~m} . \mathrm{s}^{-1}$. La valeur médiane des vitesses est de $0,3 \mathrm{~m} \cdot \mathrm{s}^{-1}$ en zone fluviale et de $0,4 \mathrm{~m} . \mathrm{s}^{-1}$ en zone de marée dynamique.

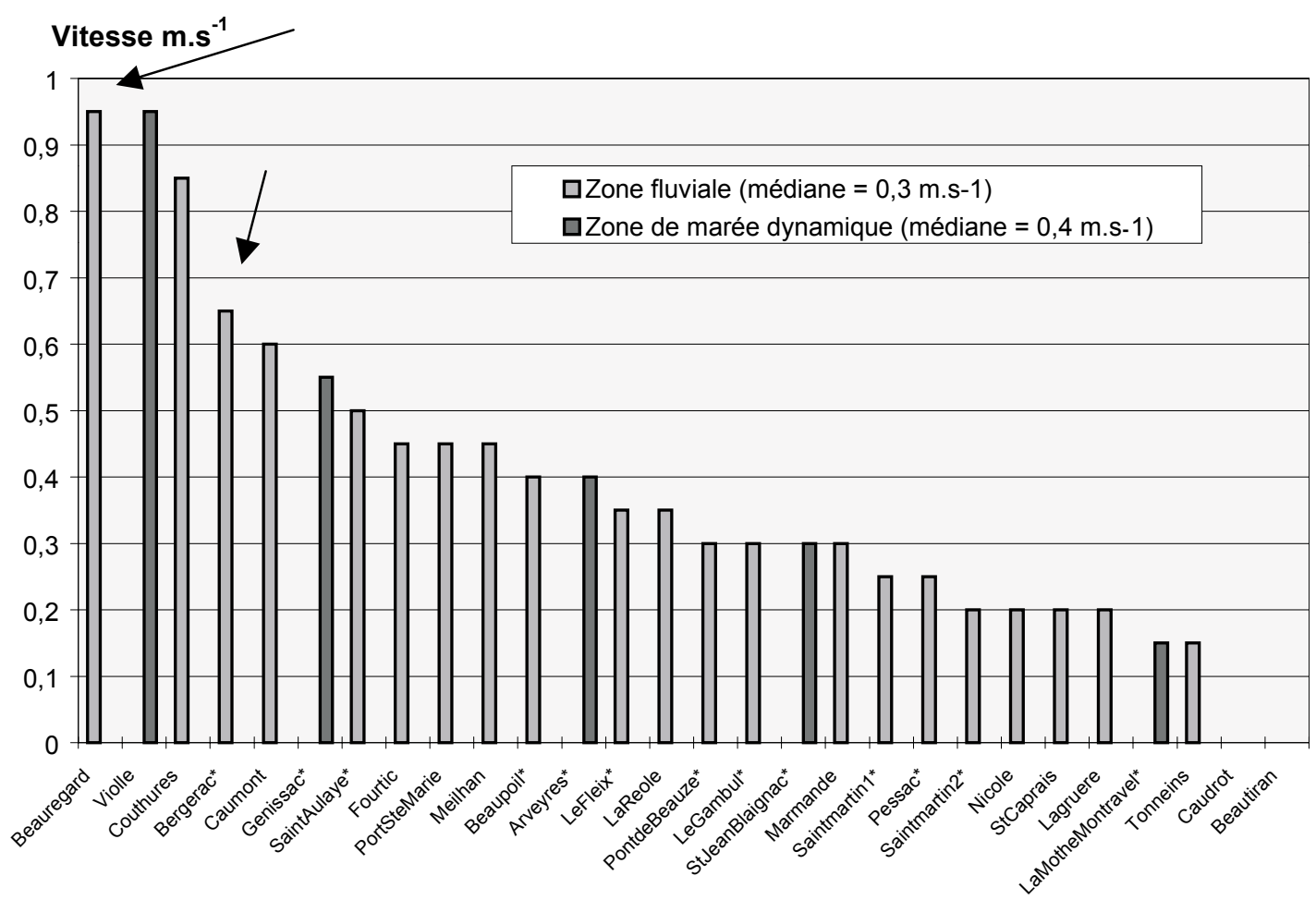

Figure 4

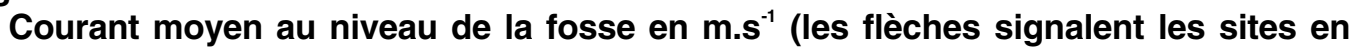
aval d'un barrage).

Figure 4

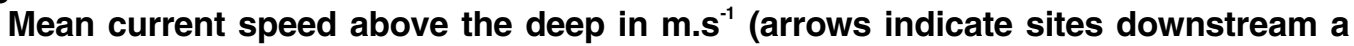
dam).

Mis à part les sites de Bergerac et de Beauregard, la profondeur maximale des sites est comprise entre 6 et $12 \mathrm{~m}$ sur la Dordogne et entre $5,5 \mathrm{~m}$ et $18 \mathrm{~m}$ sur la Garonne (Figure 5). Les valeurs médianes sont de $9 \mathrm{~m}$ en zone fluviale et en zone de marée dynamique. La valeur médiane de la différence de hauteur d'eau entre la fosse et le radier en aval (transect A) est de $6 \mathrm{~m}$ en zone fluviale et seulement de 2,5 $\mathrm{m}$ en zone de marée dynamique (Figure 6). Cette forte diminution est expliquée par la présence sur 4 sites d'une fosse peu marquée (moins de $3 \mathrm{~m}$ de différence). 


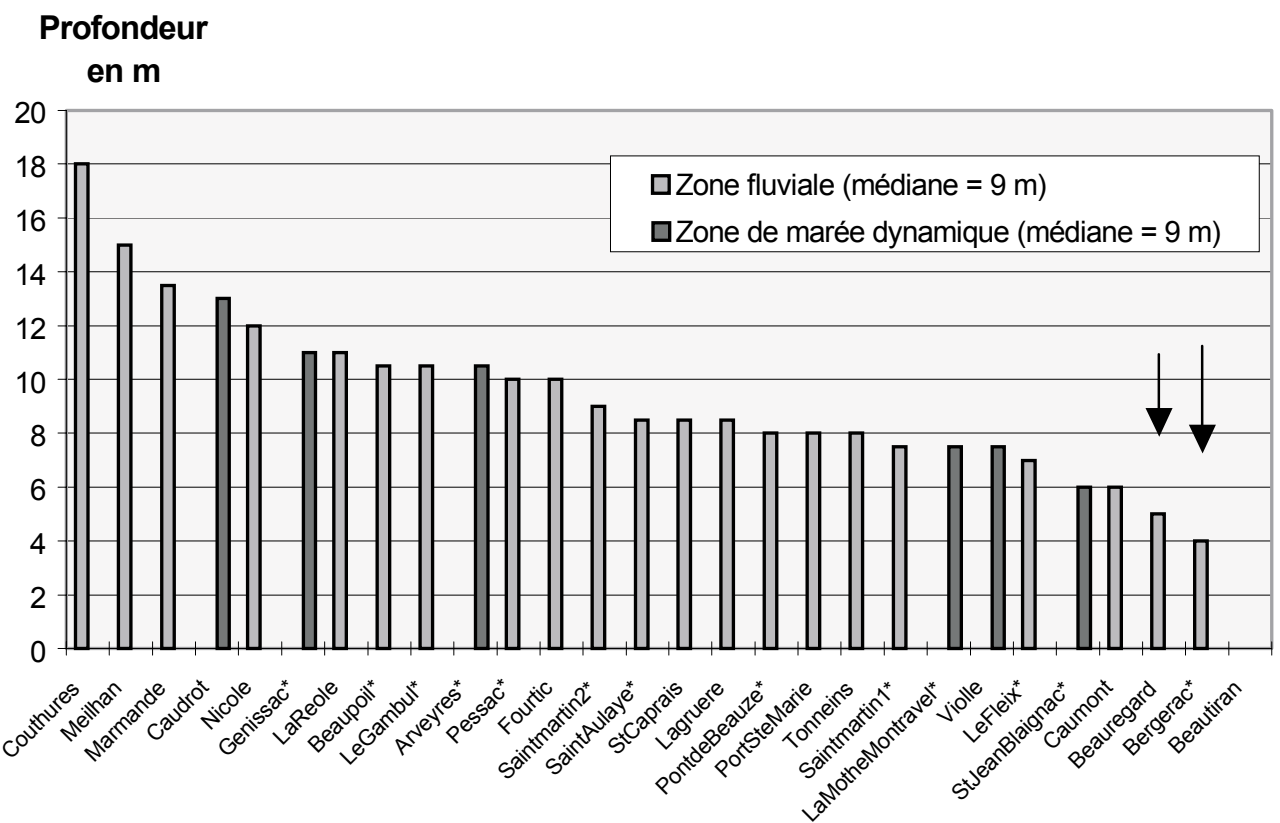

Figure 5

Profondeur maximum au niveau de la fosse en $\mathrm{m}$ (les flèches signalent les sites en aval d'un barrage).

Figure 5

Maximum depth above the deep in $\mathrm{m}$ (arrows indicate sites downstream a dam).

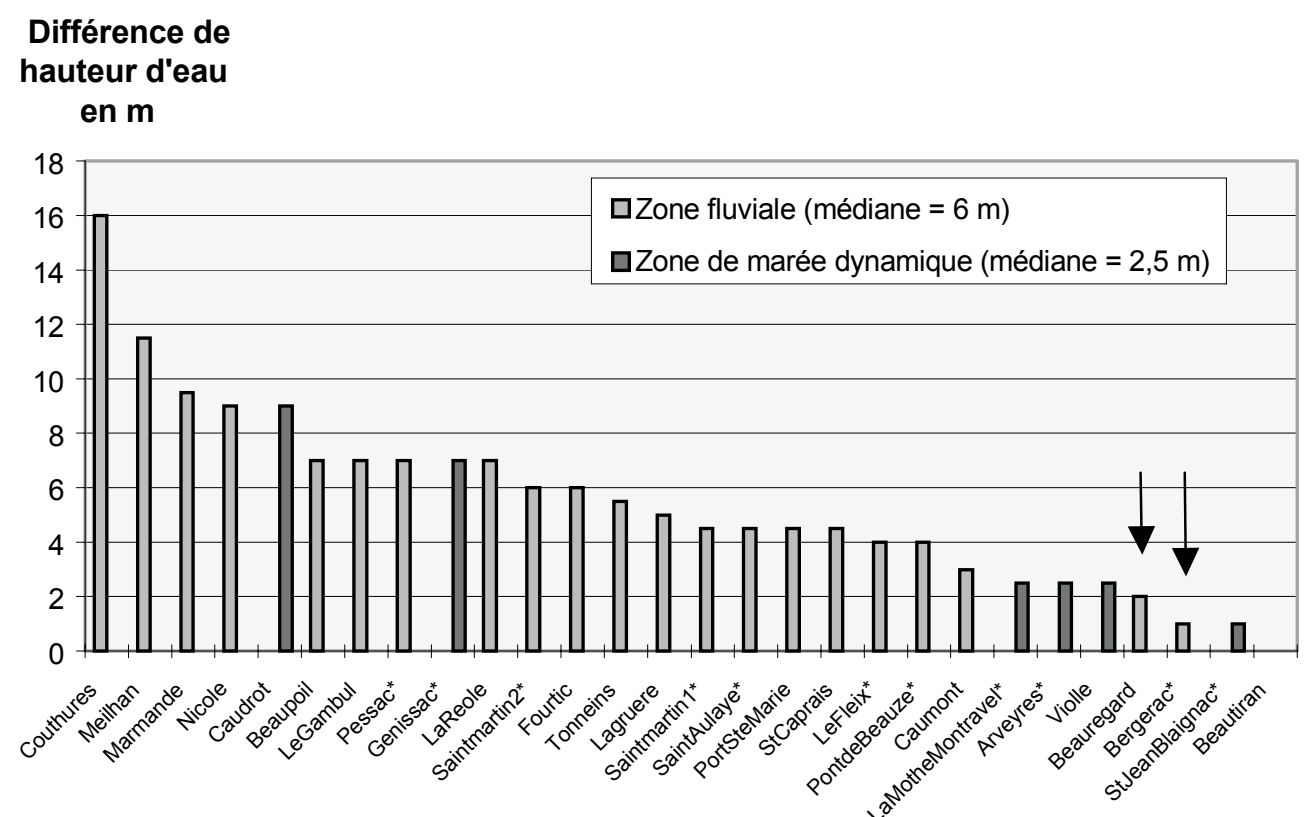

Figure 6

Différence de hauteur d'eau entre la fosse et le radier en $\mathbf{m}$ (les flèches signalent les sites en aval d'un barrage).

Figure 6

Water level difference between the deep and the riffle in $\mathrm{m}$ (arrows indicate sites downstream a dam). 
Le substrat de 23 sites est composé d'au moins $50 \%$ de cailloux. La crème de vase apparaît dans 4 des 7 sites localisés en zone de marée dynamique (Figure 7).

Les substrats des sites de la zone fluviale sont tous composés d'un mélange de cailloux et de graviers (Figure 8). La " roche mère » est le troisième constituant, apparaissant presque exclusivement dans les prélèvements des sites de Garonne.

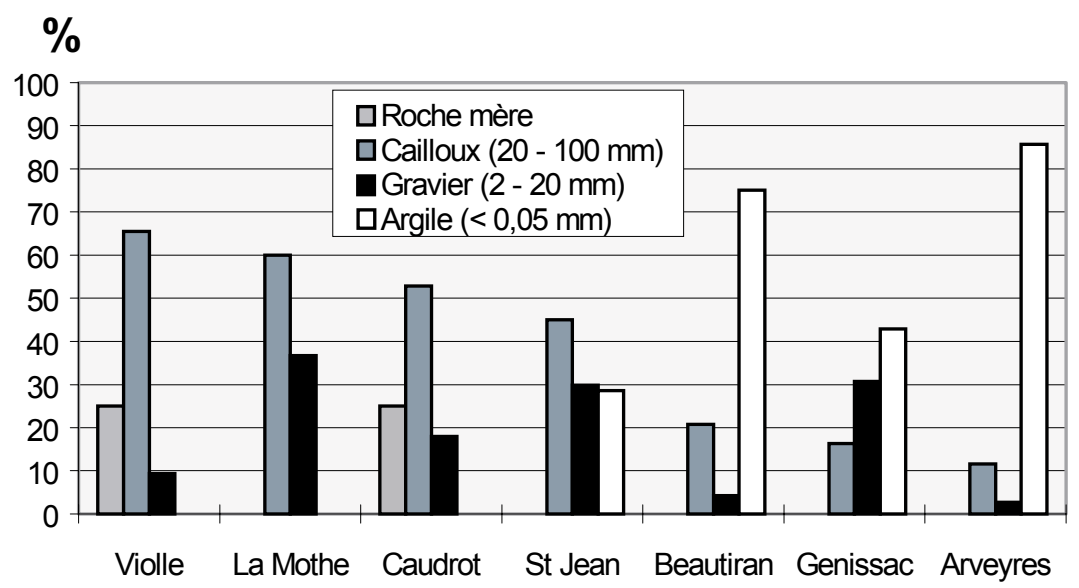

Figure 7

Composition du substrat des sites en zone de marée dynamique.

\section{Figure 7}

Substrate composition of the tidal zone sites.

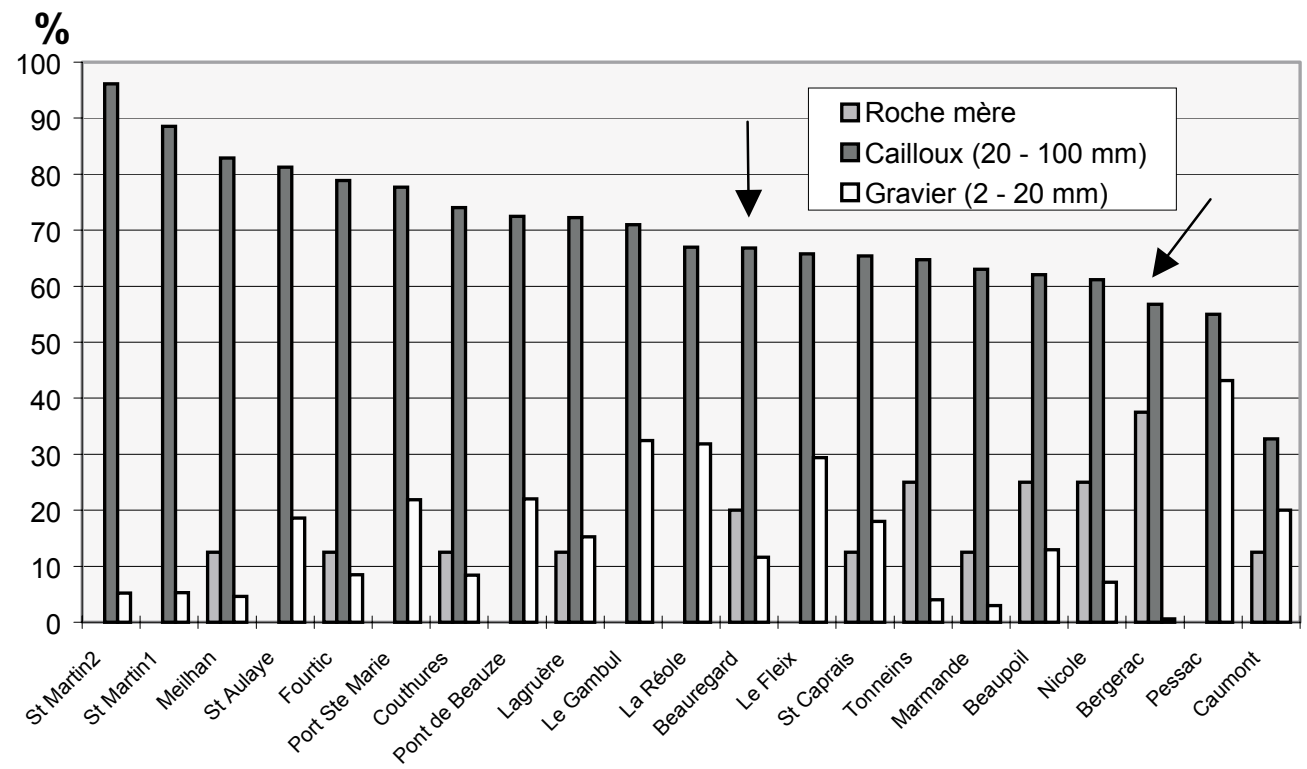

Figure 8

Composition du substrat des sites en zone fluviale (les flèches signalent les sites en aval d'un barrage).

Figure 8

Substrate composition of the fluvial zone sites (arrows indicate sites downstream a dam). 
La majorité des sites (16) ont une superficie inférieure à $20000 \mathrm{~m}^{2}$ (Figure 9). Sur les 7 sites dont la superficie est comprise entre 60000 et $150000 \mathrm{~m}^{2}, 5$ sont en zone de marée dynamique. La valeur médiane de la superficie pour les sites fluviaux est de $14000 \mathrm{~m}^{2}$ contre $76500 \mathrm{~m}^{2}$ pour les sites en zone de marée dynamique.

\section{Superficie en $\mathbf{m}^{2}$}

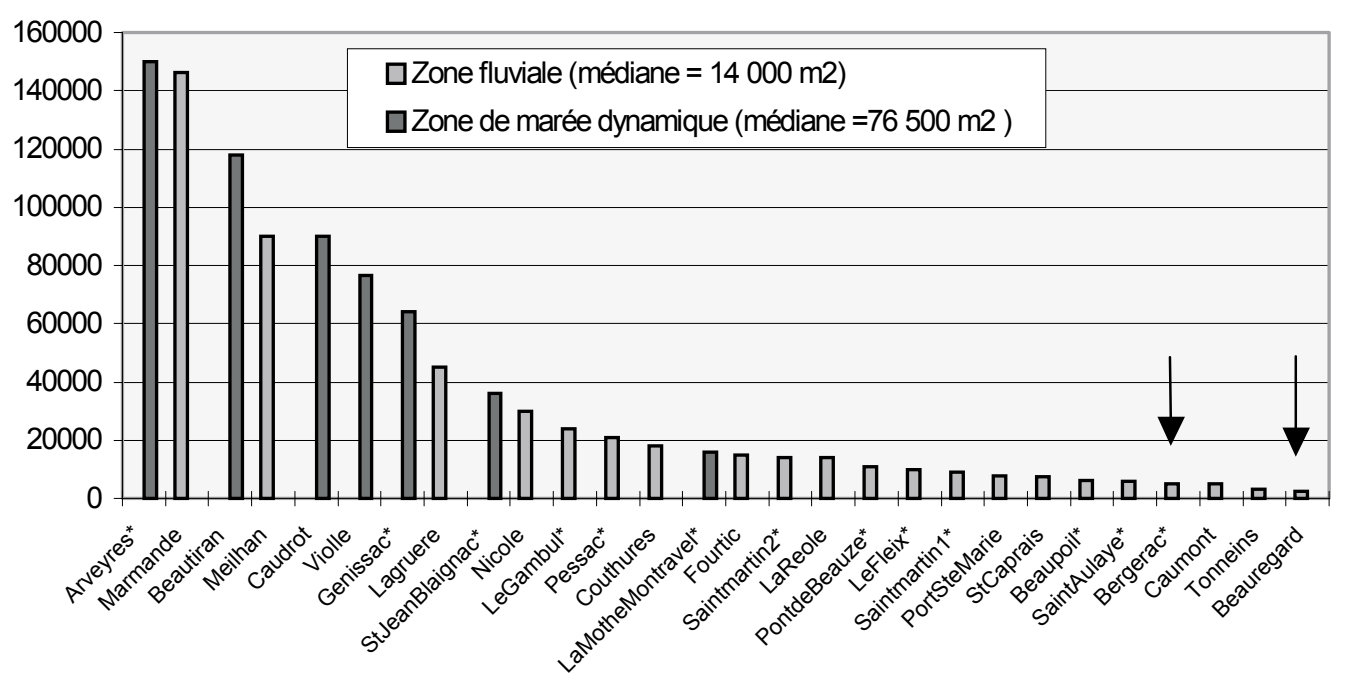

\section{Figure 9}

Superficie des sites étudiés en $\mathrm{m}^{2}$ (les flèches signalent les sites en aval d'un barrage).

\section{Figure 9}

Surface area of the studied sites in $\mathbf{m}^{2}$ (arrows indicate sites downstream a dam).

\section{DISCUSSION}

\section{Les sites en zone de marée dynamique}

Sept sites ont été étudiés dans cette zone. Seuls 2 d'entre eux, les plus en amont sur la Dordogne et sur la Garonne ne subissent pas d'inversion de courant pendant la période de reproduction de l'esturgeon.

Les valeurs médianes des critères courant moyen au niveau de la fosse et profondeur maximale de la fosse répondent aux critères de notre modèle théorique, et ne les distinguent pas des sites fluviaux. Par contre 3 paramètres les différencient nettement de ces derniers.

Hormis le site de La Mothe Montravel, la superficie des sites en zone de marée dynamique est supérieure à $20000 \mathrm{~m}^{2}$ avec une valeur médiane nettement plus élevée que celle des sites fluviaux. Ceci est expliqué par leur localisation dans la partie basse des fleuves, où la largeur est plus importante. 
La présence de crème de vase est une caractéristique de ces zones. Elle est rencontrée sur 4 sites, St Jean de Blaignac, Génissac et Arveyres sur la Dordogne, et Beautiran sur la Garonne. Selon LATOUCHE et JOUANNEAU (1994) les mouvements au sein de la crème de vase et les échanges avec la colonne d'eau sont pratiquement nuls. Cette constatation, appuyée par les données bibliographiques (KEMPINGER, 1988 ; LA HAYE et al., 1992 ; AUER, 1996), permet de mettre en doute le succès d'une éventuelle reproduction sur ces 4 sites.

Enfin la différence de profondeur entre la fosse et le radier est nettement plus faible que pour les sites fluviaux. Seuls les sites de Caudrot sur la Garonne et de Génissac sur la Dordogne présentent une fosse bien marquée (différence supérieure à $3 \mathrm{~m}$ ).

Historiquement ces sites en zone de marée dynamique étaient reconnus comme des lieux importants de pêche de géniteurs (ANONYME, 1980a, b).

Pour les sites présentant actuellement de la crème de vase, on peut émettre 2 hypothèses. Soit ces sites étaient des frayères historiques, mais les conditions hydrologiques ont été modifiées notamment par les extractions de granulats dans le lit mineur des fleuves. Ceci a eu pour conséquence d'aboutir à des zones ne répondant plus aux critères d'une frayère théorique d'esturgeon en terme de substrat. Soit ces sites n'étaient pas des frayères, mais des lieux de regroupement de géniteurs pendant la migration.

La présence de frayère en zone de marée dynamique et subissant une inversion de courant semble exceptionnelle chez les esturgeons. Néanmoins, BAIN (1997) décrit chez Acipenser oxyrinchus des frayères fonctionnelles sur la rivière Hudson, situées en zone de marée dynamique et subissant une inversion de courant. Ces informations sont intéressantes à 2 titres. La rivière Hudson présente des similarités avec la Garonne en terme de débit moyen annuel et de distance libre avant le premier obstacle infranchissable. Acipenser oxyrinchus et Acipenser sturio sont 2 espèces proches du point de vue phylogénique (BIRSTEIN et DESALLE, 1998) et de leur cycle écologique (ROCHARD et al., 1990). A la lumière de ces 2 remarques, cette information mérite donc d'être prise en considération. Nous considérons donc les 3 sites ne présentant pas de crème de vase, La Mothe Montravel sur la Dordogne, Violle et Caudrot sur la Garonne, comme des zones de frayères potentielles pour Acipenser sturio.

\section{Les sites en zone fluviale}

Les vitesses moyennes de courant, toutes inférieures à $1 \mathrm{~m} \cdot \mathrm{s}^{-1}$ au droit de la fosse, sont relativement faibles en comparaison des valeurs trouvées dans la littérature. La période de reproduction de l'esturgeon européen se situe entre mi-mai et mi-juin (MAGNIN, 1962). Les campagnes de terrain se sont déroulées durant cette période pour les sites sur la Dordogne et après pour les sites sur la Garonne. Cette période relativement tardive, lorsque les débits des fleuves sont pratiquement à leur niveau d'étiage (Figure 2), pourrait expliquer les faibles valeurs mesurées.

Les substrats sont de nature hétérogène ce qui semble être un élément déterminant pour qu'une frayère à esturgeon soit fonctionnelle. Bien que les vitesses de courant soient relativement faibles, tous ces sites ont un substrat propre, c'est à dire exempt de particules fines. Ceci est peut-être dû à l'action des crues qui permettent de " décaper » le substrat (AUER, 1996).

L'ensemble des sites de la zone fluviale sélectionnés selon notre critère profondeur, répondent également au critère de substrat et peuvent donc être considérés comme étant des frayères potentielles pour Acipenser sturio. 


\section{Les sites en aval d'un obstacle}

Bien que ces sites ne présentent pas de fosse, la profondeur maximale est du même ordre que celles relevées sur les frayères d'Acipenser fulvescens situées en aval d'un barrage hydroélectrique (GENDRON, 1988 ; PROVOST et al., 1982). Les paramètres substrat et courant répondent quant à eux parfaitement aux critères du modèle théorique de frayère à esturgeon. Etant donné que le site de Bergerac était historiquement reconnu comme une frayère pour l'esturgeon européen et du fait de leurs caractéristiques proches, nous considérons ces 2 sites comme des frayères potentielles pour Acipenser sturio.

\section{Estimation de la capacité d'accueil}

Selon HOLCIK et al. (1989), la fécondité absolue des femelles d'Acipenser sturio est plus élevée que celle des autres espèces du genre Acipenser et se rapproche de celle de Huso huso Linnaeus 1758. La superficie nécessaire pour que l'incubation des œufs de cette espèce se déroule dans de bonnes conditions de densité et d'oxygénation, est de l'ordre de $500 \mathrm{~m}^{2}$ par femelle (DERJAVINE, 1947, in VLASENKO, 1974). La superficie des 24 sites retenus comme frayères potentielles s'élève à $673000 \mathrm{~m}^{2}$. Si l'on se place dans les conditions les plus défavorables, en supposant que seulement $10 \%$ de cette surface soit utilisable par l'esturgeon, en 1996 les frayères potentielles des fleuves Garonne et Dordogne auraient pu accueillir environ 130 femelles. Selon nos connaissances de la structure de la population, seulement quelques couples de géniteurs par an sont susceptibles de venir se reproduire (ROCHARD et al., 1997). Cette capacité semble donc suffisante à moyen terme.

\section{Validité de la méthode d'approche}

Notre démarche a consisté à raisonner à partir d'un modèle théorique de frayère à esturgeon. L'application de ce modèle aux secteurs fluviaux accessibles à l'esturgeon européen a permis de sélectionner des sites où la reproduction de l'espèce semblait la plus probable. La validité de cette démarche repose sur le postulat de similarité des conditions de reproduction chez les Acipenséridés, et sur la pertinence des critères retenus.

Le principe de similarité est utilisé pour la création de frayères artificielles, dont l'efficacité a été montrée, chez l'esturgeon notamment (VLASENKO, 1974 ; GENDRON, 1988 ; DUBUC et al., 1997). Etant donné la rareté des reproductions artificielles et le niveau de nos connaissances sur les frayères d'Acipenser sturio, cette méthode d'approche était la seule possible pour recenser et caractériser l'ensemble des sites potentiels de frai sur la totalité du bassin versant. Cette démarche de caractérisation de frayères potentielles à esturgeon a été reprise pour l'estimation du potentiel d'accueil en Acipenser sturio de la rivière Odra, Allemagne (GESSNER, comm. pers.).

La profondeur minimum de $5 \mathrm{~m}$, qui était notre premier critère de sélection des sites, semble discutable car des données récentes de la bibliographie nous amènent à reconsidérer la pertinence de ce critère. Des publications sur 3 espèces nord-américaines mentionnent des zones de frai à des profondeurs comprises entre 2 et $5 \mathrm{~m}$ pour Acipenser transmontanus Richardson 1836 (SCHAFFTER, 1997), 2 et $6 \mathrm{~m}$ pour Acipenser oxyrinchus (SULAK et CLUGSTON, 1998), 1 et $6 \mathrm{~m}$ pour Acipenser brevirostrum Lesueur 1818 (KIEFFER et KINARD, 1996). Les 2 premières espèces sont de grande taille comme Acipenser sturio.

De plus, il semble établi qu'Acipenser fulvescens recherche préférentiellement des zones présentant une remontée du fond jusqu'à la surface, comme les berges, bancs dans le lit du cours d'eau, obstacles (PRIEGEL et WIRTH, 1971 ; KEMPINGER, 1988 ; LA HAYE et CLERMONT, 1996). Ce type de localisation se retrouve, d'une manière moins systématique, chez d'autres espèces (KIEFFER et KINARD, 1996). 
Etant donné la variabilité interspécifique et intra spécifique concernant ce paramètre, la notion de profondeur minimum ne semble pas un critère suffisamment pertinent pour caractériser une frayère d'Acipenséridés.

Le critère courant est également discutable. La disparité des méthodes utilisées pour enregistrer ce paramètre ne permettent probablement pas de dégager une valeur minimale requise pour considérer une frayère comme fonctionnelle.

Par contre le paramètre « différence de profondeur entre la fosse et le radier », qui n'était pas un critère de sélection, semble intéressant. II participe au principe d'hétérogénéité, de diversité d'habitat qui prévaut lors de la création d'une frayère artificielle. II semble que la notion de frayère ne doive pas se limiter au site de fixation des œufs mais s'élargir aux lieux de regroupements fréquentés par les géniteurs avant, pendant ou après la ponte étant donné la durée de présence des géniteurs sur zone (AUER, 1996) ou la fragmentation sur une ou plusieurs journées de l'acte de reproduction d'une femelle (PRIEGEL et WIRTH, 1971).

Cette réflexion débouche sur la notion de paysage d'une frayère en terme de topographie, ou de nature du fond par exemple, qui peut être une voie prometteuse pour la caractérisation des sites de frai.

\section{CONCLUSION ET RECOMMANDATIONS}

Cette étude a permis d'identifier et de caractériser 24 frayères potentielles pour Acipenser sturio. Ces sites offrent une capacité d'accueil suffisante pour le déroulement de la reproduction d'Acipenser sturio dans l'état actuel de la population. Le nombre et l'état actuel des frayères potentielles ne justifie pas la réalisation de frayères artificielles ou la réhabilitation de sites.

Si l'habitat physique des frayères potentielles d'Acipenser sturio peut être jugé satisfaisant, cela n'est pas une condition suffisante pour assurer le succès de la reproduction. De nombreuses études font ressortir que le débit est un facteur essentiel pour la migration des géniteurs. II doit se maintenir à un niveau élevé pour permettre la remontée des spécimens de grande taille (AUER, 1996) et rendre fonctionnelles les frayères dont la profondeur est la plus faible (AUER, 1996 ; VESHCHEV et NOVIKOVA, $1983,1988)$. Le succès de la reproduction est également positivement corrélé à des débits moyens précédents la période de frai (ANDERS et BECKMAN, 1993 ; PARSLEY et BECKMAN, 1994 ; SCHAFFTER, 1997 ; THIBODEAU, 1997). En cas de remontée de géniteurs, le maintien de l'intégrité du débit naturel ne pourrait être que bénéfique sur les chances de reproduction. Chez Acipenser fulvescens, NILO et al. (1997) ont montré que les conditions climatiques et hydrologiques prévalant durant l'étape larvaire semblent être déterminantes pour le recrutement. Dans cette optique le contrôle des prélèvements d'eau, à usage industriel ou agricole, réalisés dans le lit des fleuves pourrait être une mesure envisageable.

Il est également impératif de préserver l'intégrité de l'ensemble des sites répertoriés. De nombreuses menaces pèsent encore sur le lit mineur des 2 fleuves. Ce sont essentiellement les extractions de granulats qui dégradent physiquement les sites de frai en enlevant les substrats propices à l'incubation des œufs et en colmatant le fond par la décantation des particules fines remises en suspension lors du dragage. Ce type d'opération a aussi un effet négatif sur la survie des larves dérivantes (VESHCHEV, 1981). Ce sont également les aménagements de loisirs pour le tourisme fluvial, comme le surcreusement d'un chenal de navigation entre les confluences du Lot et de la Baïse avec la Garonne, à l'amont immédiat du site de Nicole. 
L'arrêté de biotope est, en France, la première mesure réglementaire à prendre pour soustraire les sites de frayères potentielles des impacts négatifs des activités anthropiques. Cette mesure de protection est prise au niveau local et peut donc être rapidement mise en œuvre. Elle concerne d'une part la préservation des biotopes nécessaires à la survie d'espèces protégées, et d'autre part, la protection des milieux contre les activités qui nuisent à leur équilibre biologique. Actuellement les sites sur la Garonne entre Beauregard et Caudrot inclus, bénéficient d'un arrêté de biotope. En revanche ceux de la Dordogne ne bénéficient pas de cette mesure de protection. II apparaît donc urgent de mettre en place de telles mesures afin de protéger les sites compris entre Bergerac et La Mothe Montravel.

Les opérations d'extraction en zones fluviales sont également interdites par le Schéma Directeur d'Aménagement des eaux Adour - Garonne.

Dans l'état actuel de nos connaissances un premier niveau de protection peut être mis en place par le contrôle des prélèvements d'eau dans les fleuves et par les arrêtés de biotope.

Une deuxième étape consistera à identifier les sites réellement utilisés par les géniteurs. Etant donné le faible effectif de géniteurs et le nombre de sites potentiels de reproduction, la récupération d'œufs ou de larves est très aléatoire voire improbable. Une solution possible consiste à effectuer le suivi des déplacements de géniteurs par télémétrie (KIEFFER et KINARD, 1996 ; MCKINLEY et al., 1998 ; SCHAFFTER, 1997). Les premiers essais techniques ont été menés en Garonne en 2000 et seront réitérés de manière à disposer d'une méthode opérationnelle lors de la remontée de géniteurs (TAVERNY, comm. pers.). Une fois un ou plusieurs sites repérés, ils devront faire l'objet d'une protection particulière. L'instrument juridique adapté correspond au classement, au niveau national, du site en réserve naturelle. En plus d'une protection renforcée, le principal avantage de cette mesure réside dans la mise en place d'une politique de gestion de la zone et d'un suivi scientifique.

Enfin, la Directive Européenne concernant la conservation des habitats naturels, de la faune et de la flore sauvages (Réseau Natura 2000) apportera peut être une solution plus globale. Les lits mineurs des 2 fleuves ont été proposés pour inscription au Réseau Natura 2000. Ainsi les zones de frayères potentielles seraient protégées, mais également les sites de repos des géniteurs et les zones d'alimentation des larves et juvéniles.

\section{REMERCIEMENTS}

Ce travail a bénéficié du soutien financier de l'Union Européenne, contrat Life $N^{\circ}$ B43200/94/754, du Ministère de l'Environnement, du Ministère de l'Agriculture et de la Pêche, des Affaires Maritimes, des Régions Aquitaine et Poitou-Charentes, des départements de la Charente-Maritime et de la Gironde et de l'Agence de l'eau Adour-Garonne. Nous tenons à remercier la Garderie du CSP, Pierre-Alain PUIVIF, Benjamin VIRY, Philippe CAMOIN et David BARBIER pour leur aide précieuse.

\section{BIBLIOGRAPHIE}

ANDERS P., BECKMAN L., 1993. White sturgeon spawning cues in an impounded reach of the Columbia River, 123-140. In : Status and habitat requirements of the white sturgeon populations in the Columbia river downstream from Mc Nary dam, Final Report, Vol II, BEAMESDERFER and NIGRO Eds., US Dept. Of Energy, Bonneville Power Administration, Division of Fish and Wildife, $421 \mathrm{p}$. 
ANONYME, 1980a. Recueil de données sur l'état des zones de pêche et des frayères potentielles de l'esturgeon (Acipenser sturio) en Dordogne du Bec d'Ambes à Castillon-la-Bataille. CTGREF, rapport préliminaire, $19 \mathrm{p}$.

ANONYME, 1980b. Recueil de données sur l'état des zones de pêche et des frayères potentielles de l'esturgeon (Acipenser sturio) en Garonne du Bec d'Ambes à Casseuil. CTGREF, rapport préliminaire, $16 \mathrm{p}$.

AUER N., 1996. Response of spawning lake sturgeons to change in hydroelectric facility operation. Trans. Am. Fish. Soc., 125, 66-77.

BAIN M., 1997. Atlantic and shortnose sturgeons of the Hudson River: common and divergent life history attributes. Environ. Biol. Fish., 48, 347-358.

BALON E., 1975. Reproductive guilde of fishes: a proposal and definition. J. Fish. Res. Board Can., 32, 821-864.

BIRSTEIN V., DESALLE R., 1998. Molecular phylogeny of Acipenserinae. Mol. Phylogenet. Evol., 9, 141-155.

BOVEE K.D.,1986. Development and evaluation of habitat suitability criteria for use in the Instream Flow Incremental Methodology. Instream Flow Information Paper 21. US Fish Wildl. Serv. Biol. Rep., 86, 235 p.

BUCKLEY J., KINARD B., 1985. Habitat use and behavior of pre-spawning and spawning sturgeon, Acipenser brevirostrum, in the Connecticut River, 111-117. In : North American Sturgeons, BINKOWSKI et DOROSHOV Eds., Junk Publishers, Dordrecht, The Netherlands, $163 \mathrm{p}$.

CASTELNAUD G., ROCHARD E., JATTEAU Ph., LEPAGE M., 1991. Données actuelles sur la biologie d'Acipenser sturio dans l'estuaire de la Gironde, 251-275. In : Acipenser, Actes du premier colloque international sur l'esturgeon, Bordeaux, 3-6 octobre 1989, WILLIOT P. ed., Cemagref, 518 p.

DENG XHONG-LING, XU YUNGAN ZHAO YAN, 1991. Analysis on Acipenser sinensis spawning ground and spawning scales below Gezhouba hydro-electric dam by the means of examining the digestive contents of benthic fishes, 243-250. In: Acipenser, Actes du premier colloque international sur l'esturgeon, Bordeaux, 3-6 octobre 1989, WILLIOT P. ed., Cemagref, 518 p.

DUBUC N., THIBODEAU S., FORTIN R., 1997. Impact de l'aménagement d'un nouveau secteur de frayère sur l'utilisation du milieu en période de fraie et le succès de reproduction de l'esturgeon jaune (Acipenser fulvescens) à la frayère de la Rivière des Prairies au printemps de 1997. Université de Montréal, Hydro-Québec, 122 p.

ELIE P., 1997. Restauration de l'esturgeon européen Acipenser sturio. Rapport final Life sturio, Etude Cemagref Bordeaux $\mathrm{N}^{\circ} 24$, ELIE Coord., $381 \mathrm{p}$.

ELIE P., ROCHARD E., TAVERNY C., 1997. Etat des frayères et proposition de restauration et de protection - Synthèse des connaissances, 129-171. In : restauration de l'esturgeon européen Acipenser sturio - Rapport final, contrat Life $\mathrm{N}^{\circ} \mathrm{B} 4-$ 3200/94/754, Etude Cemagref Bordeaux №24, ELIE Coord., 381 p.

EPIDOR, 1996. Etude préliminaire sur les frayères à esturgeon européen (Acipenser sturio) sur la Dordogne et la Garonne. $32 \mathrm{p}$.

GENDRON M., 1988. Rivière des Prairies, suivi de l'aménagement du haut-fond, résultats printemps 1988 et synthèse 1982-1988. Groupe de Recherche SeeeQ Ltée., 95 p.

JEGO S., GAZEAU C., ROCHARD E., ELIE P., 1997. Etat actuel des zones de frayères Propositions d'action de protection et de réhabilitation, 175-269. In : restauration de l'esturgeon européen Acipenser sturio - Rapport final, contrat Life N N B43200/94/754, Etude Cemagref Bordeaux №24, ELIE Coord., 381 p.

HALL J., SMITH T., LAMPRECHT S., 1991. Movements and habitat of shortnose sturgeon, Acipenser brevirostrum in the Savannah River. Copeia, 3, 695-702.

HATIN D., CARON F., FORTIN R., 1998. Recherche de géniteurs, de frayères et de juvéniles d'esturgeon noir (Acipenser oxyrinchus) dans l'estuaire fluvial du St Laurent. Ministère de l'Environnement et de la Faune, Direction de la Faune et des Habitats, $40 \mathrm{p}$.

HOLCIK J., KINZELBACH R., SOKOLOV L., VASIL'EV V., 1989. Acipenser sturio Linnaeus, 1758, 367-394. In: The Freshwater Fishes of Europe, Vol 1, Part II: General 
introduction to fishes - Acipenseriformes. HOLCIK ed., Aula-Verlag Wiesbaden, $469 \mathrm{p}$.

KEMPINGER J., 1988. Spawning and the early life history of lake sturgeon in the lake Winnebago system, Wisconsin. Trans. Am. Fish. Soc. Symp., 5, 110-122.

KIEFFER M., KINARD B., 1996. Spawning of the shortnose sturgeon in the Merrimack River, Masssachussetts. Trans. Am. Fish. Soc., 125, 179-186.

LA HAYE M., BRANCHAUD A., GENDRON M., VERDON R., FORTIN R., 1992. Reproduction, early life history, and characteristics of the spawning grounds of the lake sturgeon (Acipenser fulvescens) in Des Prairies and l'Assomption Rivers near Montréal, Québec. Can. J. Zool., 70, 1681-1689.

LA HAYE M., CLERMONT S., 1996. Localisation d'une frayère à esturgeons jaunes dans le cours inférieur de la Rivière Saint-François. Rapport Technique Enviro-Science et GLV Environnement, $15 \mathrm{p}$.

LAMONTAGNE D., GILBERT L., 1990. Etude des frayères de la rivière St Maurice en aval de la centrale de la Gabelle. G.D.G. Environnement Ltée, Hydro-Québec, 111 p.

LATOUCHE C., JOUANNEAU J.M., 1994. Etude de la dynamique de l'eau et des sédiments, 8-21. In : Livre Blanc de l'Estuaire de la Gironde, Agence de l'Eau Adour-Garonne, IFREMER, $115 \mathrm{p}$.

LECLERC P., 1987. Localisation d'une frayère à esturgeon jaune (Acipenser fulvescens) dans la rivière de l'Assomption entre Joliette et l'embouchure de la rivière Ouareau. Min. Loisir Chasse et Pêche, Québec, Direction de la Faune Aquatique, 22 p.

MAGNIN E., 1962. Recherches sur la systématique et la biologie des Acipenseridés. Ann. Sta. Centr. Hydrobiol. Appl., Paris, 9, 7-242.

MARCHANT S., SHUTTERS M., 1996. Artificial substrates collect gulf sturgeon eggs. North Am. J. Fish. Manag., 16, 445-447.

MC CABE G., TRACY C., 1994. Spawning and early life history of white sturgeon Acipenser transmontanus, in the lower Columbia River. Fish. Bull., 92, 760-772.

MCKINLEY S., VAN DER KRAAK G., POWER G., 1998. Seasonal migrations and reproductive patterns in the lake sturgeon, Acipenser fulvescens, in the vicinity of hydroelectric stations in northern Ontario. Environ. Biol. Fish., 51, 245-256.

NILO P., DUMONT P., FORTIN R., 1997. Climatic and hydrological determinants of yearclass strengh of St Lawrence River lake sturgeon (Acipenser fulvescens). Can. J. Fish. Aquat Sci., 54, 774-780.

NINUA N., 1976. Caractéristiques de l'alimentation des jeunes esturgeons, 55-60. In : l'esturgeon atlantique de la rivière Rioni, Metniesreba, Tbilissi, $121 \mathrm{p}$.

PARSLEY M., BECKMAN L., 1994. White sturgeon spawning and rearing habitat in the lower Columbia River. N. Am. J. Fish. Manag., 14, 812-827.

PARSLEY M., BECKMAN L., MC CABE G., 1993. Spawning and rearing habitat use by white sturgeons in the Columbia River downstream from McNary dam. Trans. Am. Fish. Soc., 122, 217-227.

PIROGOVSKII M., SOKOLOV L., VASIL'EV V., 1989. Huso huso (Linnaeus, 1758), 156-200. In : The Freshwater Fishes of Europe, Vol 1, Part II: General introduction to fishes Acipenseriformes. HOLCIK ed., Aula-Verlag Wiesbaden, $469 \mathrm{p}$.

PRIEGEL G., WIRTH T., 1971. The lake sturgeon: its life history, ecology and management. Wisconsin Department of Natural Resources, Publication 240-70, 19 p.

PROVOST J., FORTIN R., PATENAUDE G., PICOTTE J., HAZEL P.P., 1982. Localisation des frayères et utilisation des hauts-fonds par la faune ichtyenne, sur le site de la rivière des Prairies. Université du Québec, Montréal, Département des Sciences Biologiques, $90 \mathrm{p}$.

ROCHARD E., 1992. Mise au point d'une méthode de suivi de l'abondance des amphihalins dans le système fluvio-estuarien de la Gironde, application à l'étude écobiologique de l'esturgeon Acipenser sturio. Thèse en Biologie des Populations et Ecosystèmes, Université de Rennes I, $296 \mathrm{p}$.

ROCHARD E., CASTELNAUD G., LEPAGE M., 1990. Sturgeons (Pisces: Acipenseridae); Threats and prospects. J. Fish Biol., 37, suppl. A, 123-132. 
ROCHARD E., LEPAGE M., GAZEAU C., LAMBERT P., 1997. Suivi de la population relictuelle d'Acipenser sturio dans le milieu naturel - tableau de bord de la population, estimation de l'abondance des différentes classes d'âge, 351-374. In : restauration de l'esturgeon européen Acipenser sturio - Rapport final, contrat Life № B4-3200/94/754, Etude Cemagref Bordeaux №24, ELIE Coord., 381 p.

SCHAFFTER R., 1997. White sturgeon spawning migrations and location of spawning habitat in the Sacramento River, California. California Fish and Game, 83, 1-20.

SLIVKA A., PAVLOV A., 1982. The biological bases for changing sturgeon (Acipenseridae) fishery regulations in the Volga delta. J. Ichthiol., 22, 33-41.

SOKOLOV L., VASIL'EV V., 1989. Acipenser ruthenus Linnaeus, 1758, 227-262. In : The Freshwater Fishes of Europe, Vol 1, Part II: General introduction to fishes Acipenseriformes. HOLCIK ed., Aula-Verlag Wiesbaden, $469 \mathrm{p}$.

SULAK K., CLUGSTON J., 1998. Early life history stages of gulf sturgeon in the Suwannee River, Florida. Trans. Am. Fish. Soc., 127, 758-771.

SULAK K., CLUGSTON J., 1999. Recent advances in life history of Gulf of Mexico sturgeon, Acipenser oxyrinchus desotoi, in the Suwannee River, Florida, USA: a synopsis. J. Appl. Ichthyol., 15, 116-128.

THERRIEN J., 1998. Rapport sur la situation de l'esturgeon noir (Acipenser oxyrinchus) au Québec. Ministère de l'Environnement et de la Faune, Direction de la Faune et des Habitats, Service de la Faune Aquatique. 45 p.

THIBODEAU S., 1997. Déterminants environnementaux de la dérive larvaire de l'esturgeon jaune (Acipenser fulvescens Rafinesque) à la Rivière des Prairies, près de Montréal et potentiel d'utilisation de strontium radioactif $\left({ }^{85} \mathrm{Sr}\right)$ comme marqueur vital à court terme des stades précédant la dévalaison. Maîtrise de Biologie, Université du Québec, Montréal, $129 \mathrm{p}$.

TROUVERY M., WILLIOT P., CASTELNAUD G., 1984. Biologie et écologie d'Acipenser sturio - étude de la pêcherie. Cemagref - AGEDRA, Etude Cemagref $\mathrm{N}^{\circ} 17,79 \mathrm{p}$.

VELEZ SOTO F., 1951. Observations sur la pêche de l'esturgeon (Acipenser sturio L.) dans le Guadalquivir durant l'année 1950. Bull Fr. Piscic., 163, 49-56.

VESHCHEV P., 1981. Effect of dredging operations in the Volga River on migration of sturgeon larvae. J. Ichthyol., 21, 108-112.

VESHCHEV P., NOVIKOVA A., 1983. Reproduction of the stellate sturgeon Acipenser stellatus (Acipenseridae), under regulated flow conditions in the Volga River. J Ichthyol., 23, 42-50.

VESHCHEV P., NOVIKOVA A., 1988. Reproduction of sevryuga Acipenser stellatus, in the lower Volga. J Ichthyol., 28, 39-47.

VLASENKO A., 1974. Au sujet des frayères artificielles du fleuve Kuban. Travaux $d u$ VNIRO, 102, $29 \mathrm{p}$.

VLASENKO A., PAVLOV A., VASIL'EV V., 1989. Acipenser gueldenstaedti Brandt, 1833, 294-344. In : The Freshwater Fishes of Europe, Vol 1, Part II: General introduction to fishes - Acipenseriformes. HOLCIK ed., Aula-Verlag Wiesbaden, $469 \mathrm{p}$.

VOTINOV N., KAS'YANOV V., 1978. The ecology and reproductive efficiency of the siberian sturgeon, Acipenser baeri, in the $\mathrm{Ob}$ as affected by hydraulic engineering works. J. Ichthyol., 18, 20-29.

WILLIOT P., ROCHARD E., CASTELNAUD G., ROUAULT T., BRUN R., LEPAGE M., ELIE P., 1997. Biological characteristics of European Atlantic sturgeon, Acipenser sturio, as the basis for a restoration program in France, Environ. Biol. Fish., 48, 359-370. 
